\title{
Bacterial Colonization of Burn Wounds
}

\author{
Iman A. Hussien* Khalid A. Habib* \\ Kifah A. Jassim**
}

Received 29, November, 2011

Accepted 18, January, 2012

\begin{abstract}
:
The objective behind this work was to find out the bacteriological profile of post burn infections in wound. The study was carried out from December 2010 to February 2011 at the Burns Unit of Al -Kindy Hospital and Al-Yarmook Hospital in Baghdad. Sixty burn patients have been investigated for bacterial profile of burn wound infections. Specimens were collected in the form of wound swabs. The organisms were isolated and identified by standard microbiological methods. Antimicrobial susceptibility test has been done by ATB-PSE5 kit(BioMereiux). Pseudomonas aeruginosa 35(58.3\%) was found to be the most common isolate followed by Klebsiella pneumonae 10(16.6\%), Staphylococcus aureus 7(10\%). ,E.coli 3(5\%), Proteus merabilis 1(1.6\%), others 2(3.3\%).and sterile cultures 2(3.3\%). Antibiotic susceptibility pattren for Pseudomonas aeruginosa isolates have shown high susptibility for Amikacin 30(85.0\%), Pipracillin-Tazobactam 27(77.2\%) Piperacillin 25(71.4\%), Ciprofloxaqcin 25(71.4\%) and Colistin 24(68.5\%) and low susceptibility for Tobramycin 20(57.2\%) Gentamycin 18(51.0\%). Psudomonas was found to be resistant to most of the therapeutic agent.
\end{abstract}

Key words: burn wounds, Pseudomonas aeruginosa, Antibiotic susceptibility

\section{Introduction:}

The extensively burned or severely injured patient is at increased risk of wound infection as well as of infection of other organs as a result of various degrees of impairment of host defense mechanism [1].Burns are one of the most common and devastating forms of trauma. Patients with serious thermal injury require immediate specialized care in order to minimize morbidity and mortality. The survival rates for burn patients have improved substantially in the past few decades due to advances in modern medical care in specialized burn centers. burnrelated deaths, depending on the extent of injury, have been halved within the past 40 years [2].In patients with severe burns over more than $40 \%$ of the total body surface area (TBSA), $75 \%$ of all deaths are currently related to sepsis from burn wound infection or other infection complications and/or inhalation injury [3]. Infection of burns is common because the skin, a physical barrier against microbes, has been compromised. Furthermore, in moderate and severe burns the underlying vasculature of the skin has been damaged or destroyed and so immunity agents, such as $\mathrm{T}$ cells, cannot reach sites of infection. Accordingly, the risk of infection increases proportionately with the size of the burn [4]. The burn wound represents a susceptible site for opportunistic colonization by organisms of endogenous and exogenous origin. Patient factors such as age, extent of injury, and depth of burn in combination with microbial factors such as type and number of organisms, enzyme and toxin production, and motility determine the

*College of Science for Women \Baghdad University,

** The Central Public Health Laboratory \Ministry of Health 
likelihood of invasive burn wound infection. [5].Bacteria and fungi are the most common pathogens of burn wounds. These microbes form multispecies biofilms on burn wounds within $48-72$ hours of injury .Organisms originate from the patient's own skin, gut and respiratory flora, as well as from contact with contaminated health care environments and workers[6,7]Gram-positive bacteria are some of the first to colonize burns, followed quickly by gram-negative . Fungal infection tends to occur in the later stages after the majority of bacteria have been eliminated by topical antibiotics [4].Microorganisms routinely isolated from Burns and wounds include aerobic organisms like Pseudomonas aeruginosa,

Staphylococcus aurous, Streptococcus pyogenes, Escherichia coli, Klebsiella pneumonia, Proteus mirabilis etc., anaerobic organisms like Bacteroid fragilis, Peptostreptococcus, Propionibacterium spp., Fusobacterium spp and fungi like Aspergillus niger, Candida spp. and Zygomycetes [8]. The situation of patients with Pseudomonas aeruginosa infections is particularly problematic since this organism is inherently resistant to many drug classes and is able to acquire resistance to all effective antimicrobial drugs $[9,10]$. Pseudomonas aeruginosa has emerged as a predominant member of the burn wound flora and in the absence of topical therapy is cultured from the burn injuries of $70 \%$ patients by the third week [4].60 burn case has been investigated from burn unit of AlKindy Hospital and Al-Yarmook Hospital in Baghdad. This study will help to assess the burden of infections at the burn unit of these Hospitals and antimicrobial susceptibility testing for the most common isolate will help to formulate antibiotic policy for better management of these patients. The present study is undertaken with the following aims and objectives:

1. Finding out the bacterial profile for post burn infection in pus.

2. Evaluating the antibiotic sensitivity for the most common isolate in pus samples

\section{Materials and Methods:}

The present work includes investigation of 60 post burn infection cases admitted in burn ward of burn unit (BU) of Al-Kindy Hospital and Al-Yarmook Hospital between December 2010 and February 2011. Patients have been hospitalized in BU because of different types of burn injuries. They include; 14 (23.3\%) under 15 year-old and 46(76.7\%) over 15 year-old patients and in all patients, $18(30 \%)$ were male and $42(70 \%)$ were female. The main causes of burn injuries in this study were as follows: oil $(\mathrm{n}=24,44.4 \%)$, explosions $(\mathrm{n}=12$, $20 \%)$ boiling water $(\mathrm{n}=10,18.5 \%)$ flame $(\mathrm{n}=7,11.6 \%)$, liquid gas $(\mathrm{n}=4$, $6.6 \%)$, electrical $(\mathrm{n}=3,5.5 \%)$ The clinical samples included burn wound swabs. Wound swabs were collected aseptically and brought to the laboratory. Swabs were inoculated on Blood Agar (oxoid) and MacConkey's Agar (oxoid) and direct smears were prepared. Smears were stained by Gram's staining method. Organisms were identified by using standard biochemical tests [11] Pseudomonas aeruginosa isolates were farther identified by commercial kit API 20E (BioMerieux,France) $P$. aeruginosa isolates were stored in Brain heart infusion broth medium containing 20\% glycerol at $-20^{\circ} \mathrm{C}$. [12] .Antimicrobial Sensitivity Test (AMST) for $P$. aeruginosa isolates was done by ATBPSE5 kit (BioMerieux, France) including the following antimicrobial agents: Amikacin, Ceftazidime, Ciprofloxacin, Gentamycin, Imipenem, Meropenem, Pipracillin, Pipracillin- 
Tazobactam, Piperacillin-pyo , Tobramycin, Ticracillin ,Ticracillinepyo, Ticracillin ac .clav., Cefepim, Colistin, Cotrimoxazoland, and Ampicilin-Sulbactam.

\section{Results and Discussion:}

Infections remain the leading cause of death among patients who are hospitalized for burns. The risk of burn wound infection is directly correlated to the extent of the burn and is related to impaired resistance resulting from disruption of the skin's mechanical integrity and generalized immune suppression. (70\%) of females have been affected more than that $(30 \%)$ of males in this study. This may be because of the reason that accidental burns are more in females as they tend to spend more time near fire . Most common age group affected was 21 -
30 (Fig. 1)In this study out of 60 pus sample culture positivity was 58 (96.6 $\%$ ) while pus samples were sterile in $2(3.4 \%)$ of the cases. These results were in agreement with $[13,14]$. The absence of the growth in some samples may be because uninfected patients with aerobic bacteria or early entry to the hospital.In the present study. Pseudomonas aeruginosa 35(58.3\%) was found to be the most common isolate followed by Klebsiella pneumoniae 10(16.6\%), Staphylococcus aurous 7(11.6\%) ,Escherichia coli 3(5\%), Proteus mirabilis $1(1.6 \%)$,others $2(3.3 \%)$.and sterile cultures $2(3.3 \%)$.(Fig.2).In this study Gram negative organisms were found to be more prevalent in burn wounds, these results were in agreement with $[15,16,17,4]$.

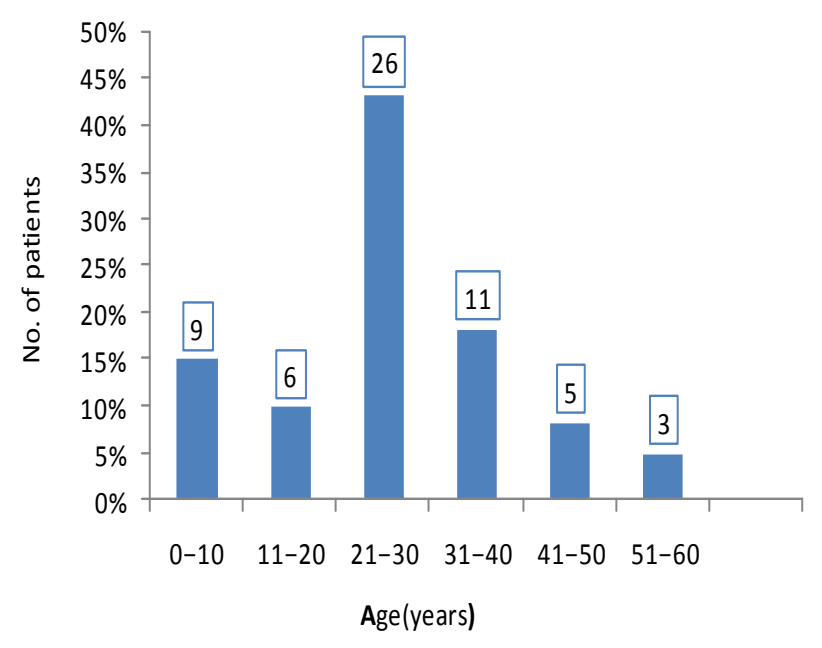

Fig1: Age distribution of the burn patients.

Many investigators have found that initially there is colonization by gram positive organisms which is replaced later by gram negative organisms. Ahmad and coworkers 2006 in their study have demonstrated that infections by gram positive organisms were more common in first 5 days of burns while gram negative organisms dominate the infection scene thereafter. 


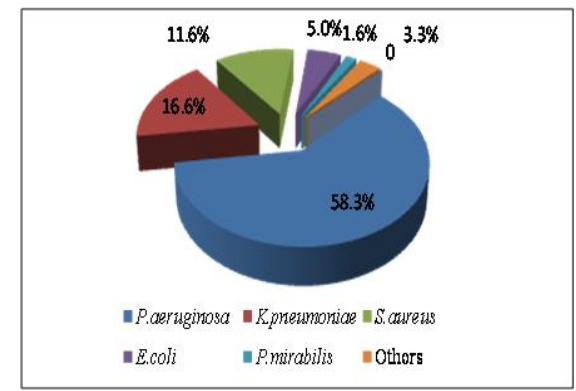

Fig.2: Microorganisms isolated from burns and their percentages.

Our finding that P.aeruginosa was the most common isolate coincides with previous reports $[8,17,18,19,20]$ this result was expected since this bacterium is resistant to many antibiotics and antiseptics.

\section{Antibiotic Susceptibility Test for P.aeruginosa}

Pseudomonas aeruginosa is inherently resistant to most Penicillins, Cephalosporins, Tetracyclines, Sulfonamides, Chloramphenicol, and Nalidixic acid. P.aeruginosa is naturally susceptible to Aminoglycosides, Antipseudomonal Penicillins, cephalosporins, quinolones, and carbapenems. However, acquired antibiotic resistance of $\mathrm{P}$. aeruginosa during treatment is a common phenomenon [21,22] This determines increased morbidity, mortality and treatment costs [23,24,25]All P.aeruginosa isolates were tested for antimicrobial susceptibilities, according to standard protocols. antimicrobials tested by means of ATB-PSE5 kit (BioMerieux) : Drug susceptibility tests show high susceptibility for Amikacin 30(85.0\%), Pipracillin-Tazobactam 27(77.2\%) Piperacillin 25(71.4\%), Ciprofloxacin 25(71.4\%) and Colistin 24(68.5\%) and low susceptibility for Tobramycin $20(57.2 \%)$ and Gentamycin 18(51.0\%)
.The results of drug susceptibility tests are showed in Table(1). The result of present study is coincides with previous study [26] that has found $P$. aeruginosa isolated is locally have shown $100 \%$ resistance for Ampicillin and Ticracillin, also this result was coincides with [27] that has found P. aeruginosa isolates from wound infections were $100 \%$ resistance to ampicillin. The findings of this study is near to [28] findings that has found 24\% P.aeruginosa isolates were resistant to pipracillin . This study also is coincides with previous study done in Iran by [35] that have found P.aeruginosa isolates from burn infections have shown low resistance for Piperacillin- Tazobactam $35.5 \%$ ,but in contrast with [26] that has found $P$. aeruginosa isolates were $100 \%$ resistant to Pipracillin, also it is in contrast with .[35] that they have found P.aeruginosa isolates shown high resistance for Piperacillin $79.1 \%$.We find the resistance of $\mathrm{P}$. aeruginosa isolates to Penicillins is reasonable since most of $\mathrm{P}$. aeruginosa strains naturally resistance to Penicillins which belong to $\beta$-lactam group, this group of antibiotics (e.g. Piperacillin , Imipenem Meropenem and Ceftazidime) inhibit peptidoglycan-assimbiling

transpeptidase located on the outer membrane of the cytoplasmic membrane.[ 33] In this study P.aeruginosa isolates have shown low resistance for ciprofloxacin $22.8 \%$ this result is coincides with previous studies [29,28] that have found the resistance of $P$. aeruginosa to this antibiotic is $4 \%$,but in contrast with [24] that they have found the resistance $\mathrm{P}$. aeruginosa isolates to this antibiotic $68 \%$. Ciprofloxacin belong to fluoroquinilons, oquinilons inhibit bacterial growth by binding to A subunit of DNA gyrase [33]. Alterations in the quinolone resistance- 
determining regions in the genes coding for DNA gyrase and topoisomerase IV, play an important role in quinolone resistance in $\mathrm{P}$. aeruginosa. [ 37]. The sensitivity to aminoglycosides (Amikacin , Tobramycin ,Gentamycin), In this study P.aeruginosa isolates have shown low resistance to this group of antibiotics $14.2 \%, \quad 34.3 \%, \quad 40 \%$ respectively.This result is coincides with previous study [27] that has found P.aeruginosa isolates have low resistance for Amikacin 20\%, also this result is coincides with [28] that she has found the resistance of P.aeruginosa isolates to Amikacin , Tobramycin and Gentamycin were $6 \%$ ,26\% and $52 \%$ respectively. Also this study is partially coincides with [ 26] that has found P.aeruginosa isolates have low resistance to Amikacin and Tobramycin but have high resistance to Gentamycin. AL-Khazali 2009 has found the resistence of P.aeruginosa isolates from burns and wounds to Tobramycin and Gentamycin were $79.6 \%, 89 . \%$ respectively these results are in contrast with the present study results. Hsu et al.,2005 have found P.aeruginosa have low resistance to Amikacin Tobramycin and Gentamycin 23\%，58\% and $48 \%$ respectively. [ 31] have found the resistance of P.aeruginosa to Amikacin and Gentamycin were $65 \%, 100 \%$ respectively. The aminoglycosides inhibit protein synthesis in bacterial cell by binding to $30 \mathrm{~S}$ subunit of the ribosome and the Aminoglycosideresistance in Pseudomonas sp. is primarily due to changes in the target enzymes and inactivation of the antibiotics [ 33 ].In this study P. aeruginosa isolates have shown high resistance for third and fourth generation of cephalosporin Ceftazidime and Cefeme $65.5 \%$, $77.2 \%$ respectively, this result is coincides with [ 26] ,also it coincides with [28] that she has found the resistance of P.aeruginosa to Ceftazidime and Cefeme 78\%,98\% respectively. But in contrast with [32] that they have found the resistance of P.aeruginosa to Ceftazidime and Cefeme $12.8 \%, 20.2 \%$, respectively. The increased prevalence of ceftazidime resistant P.aeruginosa may be related to the increased use of betalactam antibiotics such as amoxicillin and ceftazidime. Selective pressure from the use of antimicrobial agents is a major determinant for the emergence of resistant strains. The low sensitivity of P.aeruginosa isolates to carbapenems (Imipenem and Meropenem) in this study is come in coincides with [ 26] ,but in contrast with $[28,30]$ that they have found P.aeruginosa isolated from burn and wound infections have low resistance to Imipenem $0.0 \%, \quad 42.1 \%$ respectively, also this study is in contrast with $[32,35]$ that they have found the resistance of P.aeruginosa to Imipenem and Meropenem was 23.9\%, $11.3 \%$ and $15.5 \%, 37.2 \%$ respectively. Pseudomonads may develop resistance to carbapenems through combined mechanisms such as target inaccessibility, stable derepression of AmpC $\beta$-lactamase, overexpression of efflux systems and production of metallo- $\beta$-lactamases [10].The low resistance to Colistin $31.5 \%$ and high resistance to Cotrimoxazol $88.6 \%$ in this study is come in coincides with [ 30 ] that she has found the resistance of P.aeruginosa isolates to these antibiotics were $3.1 \%$, $100 \%$ respectively. But it is partially coincides with [26] that she has found P.aeruginosa isolates were resistance to both antibiotics. Anjum and Mir 2007 in their study of various nosocomial infections have found $99 \%$ of P.aeruginosa strains were resistant to Cotrimoxazol. Colistin belong to polymyxins inhibits bacterial growth 
by binding to be phospholipids in the cytoplasmic membrane, destroying its barrier function.[33]. Cotrimoxazol ( Trimethoprim and sulfamethoxazole ) these two antibiotics have a greater effect when given together than when given separately; the reason is because they inhibit DNA replication and transcription by inhibiting successive steps in the folic acid synthesis pathway, folic acid is an essential precursor in the synthesis of the DNA nucleosides thymidine and uridine. Bacteria are unable to take up folic acid from the environment thus, are dependent on their own synthesis, inhibition of the enzyme starves the bacteria of two bases necessary for DNA replication and transcription.

\section{[38].}

Table 1: Antimicrobial Susceptibility of P.aeruginosa strains isolated from burns samples.

\begin{tabular}{|l|c|c|c|}
\hline Antibiotics & $\begin{array}{c}\text { Resistant } \\
\text { strains }\end{array}$ & $\begin{array}{c}\text { Intermediat } \\
\text { strains }\end{array}$ & $\begin{array}{c}\text { Sensitive } \\
\text { strains }\end{array}$ \\
\hline FAM & $35(100 \%)$ & - & - \\
\hline TCP & $35(100 \%)$ & - & - \\
\hline TCC & $35(100 \%)$ & - & - \\
\hline TC & $35(100 \%)$ & - & - \\
\hline PIC & $10(28.5 \%)$ & - & $25(71.5 \%)$ \\
\hline PICP & $5(14.3 \%)$ & - & $30(85.7 \%)$ \\
\hline TZP & $8(22.8 \%)$ & - & $27(77.2 \%)$ \\
\hline REF & $27(77.2 \%)$ & $3(8.5 \%)$ & $5(14.3 \%)$ \\
\hline IMI & $20(57.2 \%)$ & $4(11.4 \%)$ & $11(17.1) \%$ \\
\hline MERO & $20(57.2 \%)$ & $8(22.8 \%)$ & $7(20 \%)$ \\
\hline CAZ & $22(62.5 \%)$ & $3(8.5 \%)$ & $1028.5 \%)$ \\
\hline AKN & $5(14.2 \%)$ & - & $3085.0 \%)$ \\
\hline GEN & $14(40 \%)$ & $3(8.5 \%)$ & $1851.5 \%)$ \\
\hline TOB & $12(34.3 \%)$ & $3(8.5 \%)$ & $2057.2 \%)$ \\
\hline CIP & $8(22.8 \%)$ & $2(5.7 \%)$ & $2571.5 \%)$ \\
\hline COL & $11(31.5 \%)$ & - & $24(68.5 \%)$ \\
\hline TSU & $31(88.6 \%)$ & - & $4(11.4 \%)$ \\
\hline
\end{tabular}

FAM: ampicilin-sulbactam, TCP: ticracillin-pyo, TCC: ticracillinac.clav, TC: ticracillin , PIC: piperacillin, PICP: piperacillin-pyo, TZP: piperacillin-tazobactam, REF: cefepim, IMI: imipenem, MERO : meropenem, CAZ: ceftazidime, AKN : amikacin, GEN: gentamicin,TOB : tobramycin, CIP : ciprofloxacin, COL : colistin, TSU : cotrimoxazol.

\section{References :}

1. Pruitt Jr, B. A., Lindberg, R. B. , McManus, W. F. , and Mason Jr, A. D. 1983Current Approach to Prevention and Treatment of Pseudomonas aeruginosa Infections in Burned Patients. Clin Infect Dis. 5: S889-S8 .

2. Lionelli, G. T., Pickus, E. J., Beckum O. K., , Decoursey R. L., and Korentager, R. A.. 2005. A three decade analysis of factors affecting burn mortality in the elderly. Burns 31:958-963.

3. Fitzwater, J., Purdue, G. F., Hun,J. L. and O'Keefe,G. E. 2003. The risk factors and time course of sepsis and organ dysfunction after burn trauma. J. Trauma 54:959-966

4. Church, D., Elsayed, S., Reid, O., Winston, B.and Lindsay, R. 2006. Burn Wound Infections. Clin. Microbiol. Rev., 19 (2), $403-434$.

5. Pruitt Jr,B.A., McManus, A.T., Kim, S.H., and Goodwin, C.W. 1998. Burn wound infections: Current status world. $J$. Surg...22: 135-145.

6. Chitkara Y.K. and Feierabend T.C. 1981. Endogenous and exogenousinfection with Pseudomonas aeruginosa in a burns unit, Int. Surgery $66237-$ 240.

7. Lyczak J.B., Cannon C. L., and Pier G. B2000 Establishment of Pseudomonas aeruginosa infection.. Microb. Infect. 2, 1051-1060.

8. Revathi,G., Puri, J., and Jain, B. K. 1998 Bacteriology of burns.Burns. 24:347-349.

9. Fluit, A. C., Verhoef, J.,Schmitz,F. J., and The European SENTRY Participants2001 Frequency of 
isolation and antimicrobial resistance of gram-negative and gram-positive bacteria from patients in intensive care units of 25 European university hospitals participating in the European arm of the SENTRY Antimicrobial Surveillance Program 1997-1998. Eur. J. Clin. Microbiol. Infect. Dis. 20:617-625

10. Livermore, D.M. 2002. Multiple mechanisms of antimicrobial resistance in Pseudomonas aeruginosa. Clin. Infect. Dis. 34:634-640.

11. Collee, J.G., Fraser, A.G., and Marmion, B.P., and Simmons, A. 1996.editors. Mackie and McCartney practical medical microbiology, 14th ed. London: Churchill Livingstone; p.14.

12. Lorrnce, H.G 2009. Culturing and Preserving Microorganisms, in ,Practical Handbook of Microbiology ,2nd ed,Emanuel .G.,Lorrence H. G. CRC Press Tylor and Francis Group ,Boca Raton London.

13. Husain M.T., Karim,Q.N. and Tajuri,S. 1989. Analysis of infection in a burn word. J.burns . 15(5):299-302.

14. Ingraham, J.L.and Ingraham, C.A. 2000. Inroduction to microbiology $\quad .2^{\text {nd }}$ ed.Brooks Cole.U.S.A

15. Smith, D.J Jr, and Thomson, P.D. 1992. Changing flora in burn \& trauma units. $J$. burncare Rehabil. 13:276-280.

16. Agnihotri, N., Gupta V., and Joshi, R.M. 2004. Aerobic bacterial iso isolates from burn wound infections and their antibiograms: a five-year study. Burns 30: 241-243..

17. Ahmad, M., Hussain S., Khan, I., and Malik, S.A. 2006 Pattern of Bacterial invasion in burn patients at the Pakistan institute of Medical sciences, Islamabad, Ann. of burns and fire disas..19(1): 18-21.

18. Atoyebi,. O.A., Sowemimo, G.O., and Odugbemi, T. 1992 . Bacterial flora of burn wounds in Lagos, Nigeria: a prospective study. Burns 18: 448-451. New York, , chap3.

19. Singh, N.P., Goyal, R., Manchanda, V., Das, S, Kaur, I.and Talwar, V. 2003. Changing trends in bacteriology of burns in the burns unit, Delhi, India. Burns 29: 129-132.

20. Nasser, S., Mabrouk, A. and Maher, A. 2003. Colonization of burn wounds in Ain Shams University burn unit. Burns 29: 229-233.

21. Hancock, R.E.(1998). Resistance mechanisms in Pseudomonas aeruginosa and other nonfermentative gramnegative bacteria. Clin. Infect. Dis .27(1):93-9.

22. Vitkauskienè, A., Sakalauskas, R. and Dudzevicius, V. 2003 The impact of antibiotic use on hospital-acquired pneumonia testing results. Medicina .39(3):254-9.

23. Traub, W.H., Scheidhauer, R.L., eonhard, B. and Bauer, D. (1998).Surveillance of Pseudomonas aeruginosa in intensive care units. Chemother. 44 (4) : 243-59.

24. Carmeli,Y., Troillet, N., Eliopoulos, G.M. and Samore, M.H. 1999 Emergence of antibiotic-resistant

Pseudomonas aeruginosa: comparison of risks associated with different antipseudomonal agents. Antimicrob. Agents Chemother. 43(6): 1379 -82. 
25. Ulku A., Serpil, E., Akcay M. N., Fehmi C., and Ayten, K. 2004. The time-related changes of antimicrobial resistance patterns and predominant bacterial profiles of burn wounds and body flora of burned patients. Burns.30: 660664.

26. Bdiwee, S. G. 2011 Assessment of the Resistance of the Mycobacterium tuberculosis to antibiotics and Investigating the Associated Bacteria in the Patients. Thesis Submitted to the council of the College of Science for Women, University of Baghdad in partial fulfillment of the Requirements for the Degree of Master of Science in Biology / Microbiology.

27. Al-Naddawi T.H. 2005 Study the Effect of Some Disinfectants on Some Species of Bacteria Isolated From Patients and Surgical Operating Theaters. A Thesis Submitted To The College of Science / AL - Mustansiryia University In Partial Fulfillment of The Requirements for The Degree of M Sc In Biology / Microbiology.

28. Al-Shwaikh,

R.M2006

Production and Characterization of Protease from Pseudomonas aeruginosa Isolated from Some Clinical Cases and its Relation with some Antibiotic Agents. A Thesis Submitted to the College of Science \ ALMustansiryia University. In Partial Fulfillment of the Requirements For the Degree of Doctorate of philosophy in Microbiology.

29. Qasim, K. W. 2006 Effect of Some Chemical and Physical
Factors on Pseudomonas aeruginosa Membrane permeability. A Thesis submitted to the College of Science, University of Baghdad in Partial Fulfillment of the Requirements for the degree of M. Sc Degree in Biology I Microbiology.

30. AL-Khazali K. A. 2009Resistance of Pseudomonas aeruginosa and Staphylococcus aureus isolated from Burns and Wounds infections to Antibiotics and some Disinfectants. A Thesis Submitted to the college of science \ AL-Mustansriyia University In partial fulfillment of the requirements For the Degree of MSc Degree Biology $\backslash$ Microbiology.

31. Dhar, S.,Saraf, R.,Singh K., and Raina, B. 2007Microbiological Profile of Chronic Burn Wounds among Paitients admitted in Burn Unit. JK Science 9(4):182-185.

32. Giliene. G., Pavilonis, A., and Kareiviene, L. 2007The peculiarities of Pseudomonas aeruginosa resistance to antibiotics and prevalence of serogroups. Medicina.43(1):3642.

33. Lambert ,P.A 2002 Mechanisms of antibiotic resistance in Pseudomonas aeruginosa

J.R.Soc.Med.95(41):22-26.

34. Liwimbi O. M. and Komolafe, I.O2007 Epidemeology and Bacterial Colonization of Burn Injeries in Blantyre Mlawi.Med.J. 19(1):25-27.

35. Salimi, H., Owlia, P. ,Yakhchli, B. and Lari, A. 2009Drug Susceptibility and Molecular Epidemeology of Pseudomonas aeruginosa Isolated in a Burn 
Unit. Am.J.Infect.Dis. 5(4):308313.

36. Hsu， D.I.; Okamoto , M.P. ; Murthy , R. and WongBeringer , A. 2005. Fluoroquinolone resistant Pseudomonas aeruginosa : risk factors for acquisition and impact on outcomes . $J$. Antimicrob. Chemother. 22 :17.

37. Henrichfreise B., Wiegand I., Pfister W. and Wiede B. 2007 Resistance Mechanisms of
Multiresistant Pseudomonas aeruginosa Strains from Germany and Correlation with Hypermutation. Antimicrob. Agents Chemother. 51(11): 4062-4070.

38. Laurence, D.R.; Bennett, P.N. and Brown, M.J. 1997. Clinical Pharmacology 8th ed. Churchill Livingstone, New York .

\section{الاستعمار البكتيري لجروح الحروق}

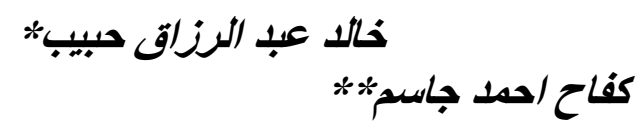

إيمان علوان حسبن*

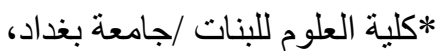

2**مختبر الصحة العام المركزي/وزارة الصحة.

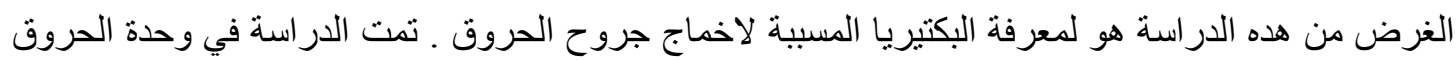

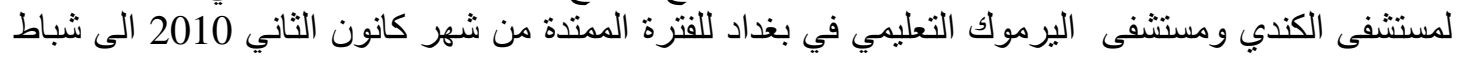

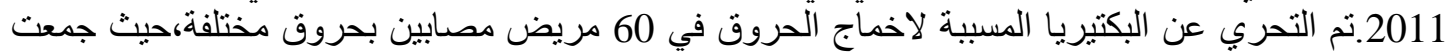

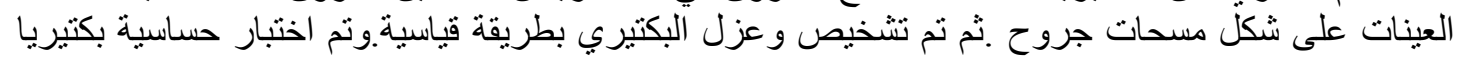

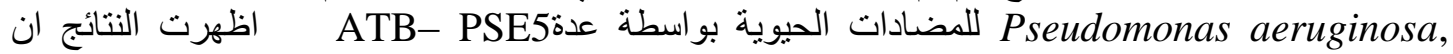
البكتيريا السالبة لصبغة غر ام هي اكثر سيادة من البكتيريا الموجبة لصبغة

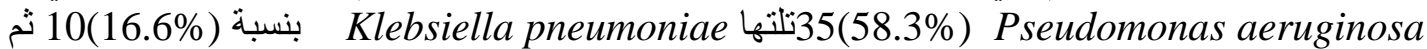
Proteus mirabilis , 3(5\%) Escherichia coli 7 7(10\%) Staphylococcus aurous

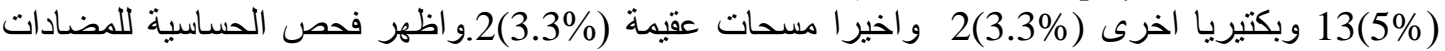

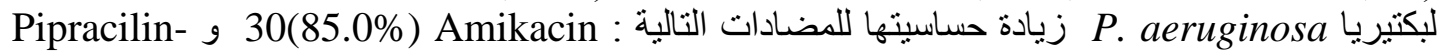
Colistin 925(71.4\%) Ciprofloxacin و Piperacillin 25(71.5\% و 27(77.2\%) Tazobactam وقلة حساسيتها لمضادي Gentamycin بنسبة (51.0\%) 18 و 20 Tobramycin (57.2\% ) .وكانت بكتيريا,Pseudomonas aeruginosa مقاومة لمعظم المضادات. 